\title{
An economic evaluation of setting up physical barriers in railway stations for preventing railway injury: evidence from Hong Kong
}

\author{
C K Law, ${ }^{1}$ P S F Yip ${ }^{2,3}$
}

- Additional appendices are published online only. To view these files please visit the journal online (http://jech.bmj.com).

${ }^{1}$ Hong Kong Institute of Asia-Pacific Studies, The Chinese University of Hong Kong, Hong Kong, PR China ${ }^{2}$ HKJC Centre for Suicide Research and Prevention, The University of Hong Kong, Hong Kong, PR China

${ }^{3}$ Department of Social Work and Social Administration, The University of Hong Kong, Hong Kong, PR China

\section{Correspondence to}

Dr C K Law, Hong Kong Institute of Asia-Pacific Studies, Room 507, Esther Lee Building, The Chinese University of Hong Kong, Shatin, New Territories, Hong Kong, PR China; lawck@cuhk.edu.hk

Accepted 2 January 2011 Published Online First 30 January 2011

\begin{abstract}
Background Setting physical barriers, for example platform screen doors (PSDs), has been proven to be effective in preventing falls onto railway tracks, but its cost-effectiveness is not known. For economic evaluation of public health interventions, the importance of including non-health factors has been noted despite a lack of empirical studies. This study aimed to investigate the effectiveness and cost-effectiveness of PSDs, which are installed in part of the Hong Kong railway system, for preventing railway injuries.
\end{abstract}

Methods Data on railway injuries from 1997 to 2007 were obtained from the railway operators. Poisson regression was used to examine the risk reduction. Two incremental cost-effectiveness ratios (ICER) were calculated to assess the cost-effectiveness based on (1) disability-adjusted life years (DALYS) only and (2) DALYS with potential fare revenue and passengers' waiting time lost due to railway circulation collapse.

Results The PSD installation has effectively reduced railway injuries (adjusted 5-year average percentage change: $-68.8 \%, \mathrm{p}<0.0001$ ) with no apparent substitution effect to the other platforms observed. To be cost-effective, the cost of gaining a healthy life year (ICER) should not exceed three times the per capita GDP (US\$74 700). The PSD installation would only be cost-effective if the loss of fare revenue and passengers' waiting time, in addition to DALY, were included (ICER: US\$65 400), while the ICER based on DALY only would be US\$77 900 .

Conclusion The challenges of complexity for economic evaluation appear in many community-based health interventions. A more extensive perspective for exploring other outcome measurements and evaluation methods to reflect a fair and appropriate value of the intervention's cost-effectiveness is needed.

\section{INTRODUCTION}

The railway, though a reliable and safe mode of public transport, poses risks of injuries and even fatalities by suicidal or accidental falls on the tracks at the railway platform edge interface. Such incidents often cause ripple effects by causing severe psychological trauma in the train driver and numerous bystanders who have witnessed an incident. ${ }^{1-3}$ In some circumstances, breakdowns of railway circulation for several hours are implicated, which may entail after-effects for large parts of the system. ${ }^{1}$ How to prevent falls on railway tracks has become an important public health issue.

The existing literature suggests that setting up physical barriers to restrict passengers' access to railway tracks is a highly effective way to mitigate the risk, ${ }^{13-9}$ such as by means of platform screen doors (PSDs) along the platform edge interface in stations. ${ }^{2}$ As a social investment for public health intervention, such an installation programme requires a rigorous assessment to evaluate if all resources used can bring the highest net benefit to society.

Economic evaluation in health economics compares the costs and health consequences of alternative courses of action to inform decisionmakers in an explicit, quantitative and systematic way to make judgements under the principle of 'value for money'. ${ }^{11}$ Cost-effectiveness has been found to be an important issue in health topics. While there have been a considerable number of guidelines on such analyses in clinical settings, its application on public health interventions is still limited and controversial. This has been mainly attributed to lack of understanding, mistrust of methods used and investigators' motives. ${ }^{10} 12 \quad 13$ The gap between scientific research and on-site implementation has further introduced a sense of irrelevance and illusion of such analyses. ${ }^{10}{ }^{14-16}$

The WHO's Choosing Interventions that are Cost Effective Project (WHO-CHOICE) has developed new methods to assess the cost-effectiveness of interventions. ${ }^{17-25}$ They propose that all economic assessments should be undertaken from a societal perspective to facilitate policymakers in determining the most beneficial way for the use of scarce resources. ${ }^{26} \mathrm{~A}$ list of guidelines and criteria on classification and measurement of costs, assessment of interventions' effectiveness, formulation of cost-effectiveness, and interpretation of results and uncertainty are also proposed for enhancing comparability among different public health interventions to ensure the results are relevant for practical policy decisions. ${ }^{17}{ }^{19}$ The WHO guidelines on generalised cost-effectiveness analysis have provided a more transparent, extensive and comprehensive theoretical framework with more relevance to rebuild the confidence towards the technique for improving health. In practice, however, more considerations and adaptations may be needed, especially on evaluation of health outcomes of public health interventions. ${ }^{27}$

The WHO guidelines suggest measuring health changes at the individual level and summing them up to capture the societal effect. ${ }^{28}$ The assumptions behind this method, however, may not hold when it is applied to deal with the multiple effects of an intervention that involves a complex system in which any change due to the intervention is likely 
to have diverse, far-reaching and non-linear effects on the overall community health. ${ }^{27-29}$

The railway is one of the most prominent means of public transport worldwide; falls onto railway tracks do not often happen but have severe impacts on not just the death, but also the railway company, the driver, passengers and witnesses of the person-train collision. ${ }^{1-3} 30$ To measure the effectiveness of installing physical barriers (eg, PSDs) to restrict passengers' access to railway tracks in such a complex situation, the use of traditional economic evaluation, which mainly focuses on improvement in health as the final outcome, may have a high risk of omitting other material non-health outcomes and thus underestimate the cost-effectiveness. Instead, more collective and deliberative methods that elicit societal values are needed to evaluate the economic efficiency of public preventive measures. 272831

The present study was designed to fill the gap in economic evaluation on public health preventive measures in a railway system, taking societal values into consideration. This paper specifically aims to (1) evaluate the effectiveness of installing PSDs at the underground railway for preventing suicidal and accidental falls on railway tracks from a societal perspective; (2) assess and compare the cost-effectiveness of the intervention by (a) using the traditional WHO-CHOICE approach and (b) a modified framework based on WHO-CHOICE but incorporating other related non-health outcomes.

\section{METHODS \\ Study design and setting}

The present study examines the effectiveness and cost-effectiveness of PSDs for preventing railway injuries in Hong Kong. Information related to falls onto railway tracks over the study period of 11 years from 1997 to 2007 was made available from the Safety Office of the Mass Transit Railway Corporation Limited (MTRCL) through the Transport and Housing Bureau of the Hong Kong Special Administrative Region government. The patronage figures, the cost and the schedule of the PSD installation programme over the period were made available from the MTRCL. Information on per capita gross domestic product (GDP) was made available from the Census and Statistics Department of Hong Kong.

Overall, the nature (ie, suicidal or accidental) and outcome (ie, fatal or non-fatal) of incidence were made available from the MTRCL's dataset, as safety officers at stations would conduct a preliminary investigation shortly after the incidence. For cases with fatal outcome, we verified the external cause of death for each incidence with the Coroner's Court of Hong Kong. Hong Kong has a very reliable coronial system with good quality of mortality data. Under the ICD category, all reportable deaths with external cause code as E958.0 (for cases from 1997 to 2000) and X81 (2001 and onwards) that satisfied the definition of 'intentional self-harm by jumping or living in front of a moving object' were classified as railway suicide. ${ }^{32}{ }^{33}$ Those coded as E805 (from 1997 to 2001) and V81.4 (2001 and onwards) were defined as accidental deaths. ${ }^{32} 33$

Over the study period (1997-2007), the railway system of Hong Kong was managed by two different operators: the MTRCL and the Kowloon-Canton Railway Corporation (KCRC). In 2002, the MTRCL started to retrofit PSDs at all the platforms in the 30 underground stations. ${ }^{34}$ Most of the platforms of the busiest station were sealed with PSDs in 2002, and the whole installation work was completed in 2005. The incidental benefit of the installation programme has been a much safer environment that prevent falls onto railway tracks, in addition to the primary purpose of energy conservation by keeping the cool air in the stations. ${ }^{2}$ On the other hand, the eight MTRCL elevated stations and the 22 KCRC stations remained unsealed. This indirectly created a quasi-experimental setting for the present study to assess the effectiveness and costeffectiveness of the PSDs for preventing railway injuries. The period 1997-2001 was taken as the preinstallation period and 2003-2007 as the postinstallation period, while 2002, the installation year, was excluded.

\section{Effectiveness}

Measures at both individual and societal levels were adopted for studying the effectiveness of PSDs in preventing railway injuries: (1) the number of suicidal and accidental falls, (2) disability-adjusted life years (DALYs), (3) the overall passengertime and (4) the fare revenue saved after PSD installation. Population health effects due to the installation of PSDs for the prevention of suicidal and accidental falls were separately assessed using Poisson regression models implemented in SAS GENMOD software (see online appendix 1 for details).

The potentially avertable disease burden for suicidal and accidental falls on railway tracks at the PSD-sealed platforms was quantified using the DALY. DALY is a time-based, composite measure of health status. It is the sum of the years of life lost (YLL) due to premature mortality and also the years lost due to disability (YLD). ${ }^{35-37}$ To calculate the YLL, we multiplied the number of deaths at each age by the corresponding global standard life expectancy. For estimating the non-fatal health outcome (ie, YLD), it was calculated from an incidence perspective, in which the incidence, average duration of disability and associated disability weights were multiplied according to the severity conditions reported from the MTRCL. Details of DALY estimation for each severity condition can be found in appendix 2 (online).

The consequence of every incident at the railway platform edge interface should not simply be a matter of health changes at the individual level. From a broader socio-economic perspective, the subsequent loss arisen from breakdowns of railway circulation due to railway injuries must be taken into account. Therefore, in addition to quantifying the health effects of PSDs in terms of number of incidents and DALY, we also estimated the overall passenger time, by multiplying the total years saved from service disruption by the passenger volume over the period, and the fare revenue that has been saved due to service disruption at formerly MTRCL net after the installation of PSD.

\section{Costs}

Two billion Hong Kong dollars (US\$256.4 million) was spent for the PSD installation programme, according to MTRCL. ${ }^{38}$ To provide a reliable estimate to allocate the amount of costs incurred over the whole installation period from 2002 to 2005, we used the percentage of completion method, in which the expenses can be attributed to the proportion of work completed. ${ }^{39}$ All the costs were converted from Hong Kong dollars (US\$ $1=\mathrm{HK} \$ 7.8$ ) and expressed in 2002 US dollars.

\section{Cost-effectiveness}

To evaluate whether the installation of PSDs is economically efficient from a societal perspective; we estimated the incremental cost-effectiveness ratio (ICER) for the intervention by two approaches. First, we followed the recommendations of WHO-CHOICE for generalised CEA, if applicable. The guidelines propose that all economic assessments should be undertaken from a societal perspective, which facilitates policy to get 
the most beneficial way for the use of scarce resources available to a society. ${ }^{26}$

In the WHO-CHOICE approach, we used the avertable DALYs as the only outcome measure for the intervention's effectiveness. As the WHO-CHOICE guidelines have no specific information on the useful life of PSD, we followed the MTRCL's accounting policy on Plant and Equipment that assumed a useful life of 25 years for PSDs. ${ }^{40}$ All future costs and avertable life years were discounted continuously at an annual rate of $3 \% .{ }^{11} 41$

On the basis of the WHO-CHOICE guidelines, we proposed a modified framework for calculating the ICER by incorporating other non-health-related effects. In this approach, the programme's effectiveness is synthesised by three separate components: (1) the avertable DALYs from railway injuries; (2) the avertable passenger's waiting time due to the breakdown of railway circulation; and (3) the corresponding financial savings from the loss of fare revenue. The ICER equals the net installation cost for the railway operator (subtracting the discounted installation cost by the discounted financial savings from the loss of fare revenue) divided by the total discounted life years gained from both the potential victims and the passenger time. Mathematically, the formula for ICER can be written as follows:
Kong, yielding a toll of 402 casualties (154 suicidal and 248 accidental). Overall, the case death rate of suicidal injuries was far higher at $50 \%$ than that of accidental injuries at $0.8 \%$ (two out of 248). Also, among those incidents with non-fatal outcomes, those who fell by accident usually had a less severe condition. Only 33\% (N=82) had a serious condition, while the same figure for non-fatal suicidal injuries was higher at $67.5 \%$ $(\mathrm{N}=52)$ over the period.

As shown in table 1, the total number of casualties at the formerly MTRCL net is reduced by $66.8 \%$ from 202 before the installation programme (1997-2001) to 67 after the programme (2003-2007). In contrast, the corresponding figures at the formerly KCRC net have been maintained at a fairly stable level of 25 over the entire study period. When separately analysed by nature and outcome of the incidence before and after the installation programme, we observed that the number of fatal suicidal falls has dropped the most at $78.9 \%$ from 38 to 8 . The number of accidental falls has been reduced by $67.9 \%$ (from 131 to 42 ), while the number of non-fatal suicidal falls has also been reduced at $48.4 \%$.

Poisson regression analyses confirmed that the presence of PSDs has effectively reduced the risk at the platform edge

$$
\text { ICER }=\frac{\text { discounted installation cost }- \text { discounted savings from fare revenue }}{\text { discounted DALYs averted }+ \text { discounted passenger time }}
$$

For the classification and evaluation of cost-effectiveness, we followed the recommendation proposed by Murray and colleagues $^{42} 43$ on the basis of the WHO's Commission on Macroeconomics and Health. They define interventions that save a healthy life year at a cost below the state's GDP per head as very cost-effective; those averting at a cost between one and three times the per capita GDP are cost-effective, and the rest are not cost effective. ${ }^{43}$

\section{RESULTS \\ Risk reduction at the platform edge interface after the PSD installation}

Over the 11-year period from 1997 to 2007, a total of 397 incidents of railway injury were identified and reported in Hong interface of the formerly MTRCL net with an adjusted 5-year average percentage change (APC) of $-68.8 \%(p<0.0001)$ (see table 2). Meanwhile, the change did not appear to affect the risk to other unsealed railway platforms, as the corresponding figure at the formerly KCRC net did not show a significant rise $(p=0.5688)$. Finally, within the formerly MTRCL net, it is worth noting that the overall risk reduction was not proportionately distributed according to the nature and outcome of the incidence. Overall, the risk of fatal suicidal falls was reduced the most with a 5 -year APC of $-80.6 \%(p<0.0001)$, which was followed by accidental falls at $-69.5 \%(p<0.0001)$. Finally, the risk of non-fatal suicidal fall also recorded a significant reduction with APC of $-52.6 \%(p=0.0126)$, even though it changed the least when compared with the other two categories.

Table 1 Number of suicidal and accidental fall on railway tracks in Hong Kong by outcome and railway network, pre- and postinstallation period*

\begin{tabular}{|c|c|c|c|c|c|c|}
\hline \multirow[b]{2}{*}{ Type } & \multirow[b]{2}{*}{ Outcome } & \multirow[b]{2}{*}{ Condition } & \multicolumn{2}{|c|}{ Mass Transit Railway Corporation Limited } & \multicolumn{2}{|c|}{ Kowloon-Canton Railway Corporation } \\
\hline & & & Preinstallation & Postinstallation & Preinstallation & Postinstallation \\
\hline \multirow[t]{5}{*}{ Suicidal } & Fatal & & 38 & $8 \dagger$ & 13 & 15 \\
\hline & Non-fatal & & 33 & 17 & 11 & 12 \\
\hline & & Serious & 27 & 12 & 5 & 6 \\
\hline & & Minor & 2 & 0 & 1 & 1 \\
\hline & & None apparent & 4 & 5 & 5 & 5 \\
\hline \multirow{5}{*}{ Accidental } & Non-fatal & & 131 & 42 & 22 & 25 \\
\hline & & Serious & 41 & 16 & 5 & 11 \\
\hline & & Minor & 57 & 18 & 14 & 7 \\
\hline & & None apparent & 33 & 8 & 3 & 7 \\
\hline & Overall & & 131 & 42 & 22 & 27 \\
\hline Both & Fatal & & 38 & 8 & 13 & 17 \\
\hline
\end{tabular}

\footnotetext{
*The preinstallation period refers to 1997-2001, and the postinstallation period refers to 2003-2007.

†Subject to reporting delay, one case of a fatal suicidal fall on the railway at formerly Mass Transit Railway net was reported from the Coroner's Court of Hong Kong after the publication date of Law et al. ${ }^{2}$
} 
Table 2 Results of age- and gender-adjusted slope coefficients from the Poisson regression model and the corresponding average percentage change of suicidal and accidental falls on railway track in Hong Kong between the pre- and postinstallation period*

\begin{tabular}{llllcc}
\hline Type & Outcome & $\begin{array}{l}\text { Railway } \\
\text { system }\end{array}$ & $\boldsymbol{\beta}$ & $\begin{array}{l}\text { Average } \\
\text { percentage } \\
\text { change (\%) }\end{array}$ & p Value \\
\hline Suicidal & Fatal $\dagger$ & MTRCL & -1.64 & -80.6 & $<0.0001$ \\
& & KCRC & 0.08 & 8.8 & 0.824 \\
& \multirow{2}{*}{ Non-fatal } & MTRCL & -0.75 & -52.6 & 0.0126 \\
& & KCRC & 0.02 & 1.5 & 0.9713 \\
& Overall & MTRCL & -1.12 & -67.5 & $<0.0001$ \\
& & KCRC & 0.05 & 5.5 & 0.85 \\
Accidental & Overall & MTRCL & -1.19 & -69.5 & $<0.0001$ \\
& & KCRC & 0.18 & 19.6 & 0.5331 \\
Both & Overall & MTRCL & -1.16 & -68.8 & $<0.0001$ \\
& & KCRC & 0.11 & 12.1 & 0.5688
\end{tabular}

*The preinstallation period refers to 1997-2001, and the postinstallation period refers to 2003-2007.

tSubject to reporting delay, one case of a fatal suicidal fall on the railway at formerly Mass Transit Railway Corporation Limited (MTRCL) net was reported from the Coroner's Court of Hong Kong after the publication date of Law et al. ${ }^{2}$

KCRC, Kowloon-Canton Railway Corporation.

\section{Socio-economic consequence of PSD installation}

As the absolute risk at the platform edge interface is lowered, we found an overall reduction in terms of DALYs and service disruption at the formerly MTRCL net upon the presence of PSD. As shown in table 3, the overall DALYs over the postinstallation period (2003-2007) have been reduced by more than $75 \%$ from 1168 to 287 years, which implies a total of 881 healthy life years averted after the PSD installation. Also, at a macroeconomic level, the presence of PSD has significantly shortened the period of service disruption by $69.4 \%$ (or $1208 \mathrm{~min}$ ) from 1739 to 531, which indicates a saving of 201 passenger-years during the same period. In line with the results from Poisson regression, the overall benefits from setting up PSDs mainly go to the prevention of fatal suicidal falls, which contributed to $76.7 \%$ of the total averted DALYs and $64 \%$ of the reduced time of service disruption.

\section{Cost-effectiveness of setting up PSDs from a public safety perspective}

Table 4 shows a summary of the cost-effectiveness analysis, which includes the overall discounted costs, effects and ICERs of

Table 3 Estimated disability-adjusted life years and delay of railway services at the formerly Mass Transit Railway Corporation Limited net by type of incidence, preinstallation and postinstallation period*

\begin{tabular}{|c|c|c|c|c|}
\hline \multirow[b]{2}{*}{ Outcome measure } & \multirow{2}{*}{$\begin{array}{l}\text { Type of } \\
\text { incidence }\end{array}$} & \multicolumn{2}{|l|}{ Period } & \multirow{2}{*}{$\begin{array}{l}\text { Period } \\
\text { change }\end{array}$} \\
\hline & & Preinstallation & Postinstallation & \\
\hline \multirow{6}{*}{$\begin{array}{l}\text { Disability-adjusted life } \\
\text { years (person-years) }\end{array}$} & Fatal & & & \\
\hline & Suicidal & 801 & 125 & 676 \\
\hline & Non-fatal & & & \\
\hline & Suicidal & 144 & 63 & 81 \\
\hline & Accidental & 223 & 99 & 124 \\
\hline & All & 1168 & 287 & 881 \\
\hline \multirow[t]{6}{*}{ Delay of service ( $\mathrm{min}$ ) } & Fatal & & & \\
\hline & Suicidal & 937 & 161 & 776 \\
\hline & Non-fatal & & & \\
\hline & Suicidal & 453 & 238 & 215 \\
\hline & Accidental & 349 & 132 & 217 \\
\hline & All & 1739 & 531 & 1208 \\
\hline
\end{tabular}

*The preinstallation period refers to 1997-2001, and the postinstallation period refers to 2003-2007.
Table 4 Estimated costs, effectiveness and incremental costeffectiveness ratios for setting up platform screen doors at formerly Mass Transit Railway Corporation Limited net by scenario

\begin{tabular}{lrr}
\hline & \multicolumn{2}{c}{ Evaluative approach } \\
\cline { 2 - 3 } Item & Traditional & \multicolumn{1}{c}{ Modified } \\
\hline Costs & 237748900 & 237748900 \\
$\quad$ Installation (US\$) & & 7897200 \\
$\quad$ Less: savings of fare lost (US\$) & 237748900 & 229851700 \\
$\quad$ Net cost (US\$) & & \\
Effectiveness (person-year) & 3053 & 3053 \\
$\quad$ Disability-adjusted life years & & 464 \\
$\quad$ Passenger-time & 3053 & 3517 \\
$\quad$ Overall effectiveness (person-year) & 77874 & 65354 \\
$\begin{array}{l}\text { Incremental cost-effectiveness ratios } \\
\text { (US\$ per person-year) }\end{array}$ & & \\
$\begin{array}{l}\text { Minimal useful life-years for platform screen } \\
\text { door required in achieving a cost-effective }\end{array}$ & 27 & \\
public health intervention (years) & & \\
\hline
\end{tabular}

setting up PSDs at different scenarios. The installation cost of PSDs amounted to US\$ 237 million after adjustment of price and discounting, which is slightly smaller than the reported cost at US\$ 256.4 million.

In the traditional approach, the installation of PSDs would save about 3100 DALYs, and the programme would have an ICER of US\$ 77900 per year, which is 3.13 times the per capita GDP, marginally exceeding the threshold of a cost-effective intervention at US\$74700 (three times the per capita GDP). The intervention only becomes cost-effective when we allow a longer expected useful life of PSDs of two additional years to 27 years, by which the amount of avertable DALYs would increase by $5.2 \%$ to 3200 years, and this would make a reduction of ICER to US\$74000 per life-year.

In contrast, in the modified approach where the savings of fare revenue and passenger waiting time are taken into account, the results are different. In addition to the avertable DALYs, we also estimated that the installation of PSDs would save about US\$7.9 million for the railway operators from the loss of fare revenue and 460 life-years from passengers' waiting time. These factors would reduce the ICER to US\$65 400 per life-year, which is 2.62 times the per capita GDP. The intervention would remain cost-effective, even if the useful life of PSD were to be shortened to 21 years. Furthermore, the cost saving of energy conservation due to PSD has not been included in the calculation. The management of MTRCL claimed that the cost saving arising from setting up the PSD to keeping the cool air inside the station without losing into the tunnel is the original purpose of the measure. $^{38}$

\section{DISCUSSIONS}

To the best of our knowledge, this is the first evaluative study worldwide to assess the economic efficiency of setting up PSDs at the railway platform interface for public safety purposes. Following the intervention, the overall risk of both suicidal and accidental falls showed a significant reduction. In addition, our economic assessment has revealed that the installation of PSDs is cost-effective from a larger societal perspective in which the interest of the victims, the railway operators and passengers are all taken into consideration. The present study demonstrates the analysis of a complex intervention delivered at the population level using an economic evaluation framework.

The challenges of complexity appear in many public health interventions. Many broad and community-based health 
interventions often involve activities outside the formal health sector. Additional costs and benefits beyond those originally envisaged in the research protocol may be induced which would directly affect the interpretation of the results. ${ }^{28} \mathrm{~A}$ more extensive perspective for data collection to identify and capture signs of other non-linear changes, unintended consequences and multiplier effects has been suggested. ${ }^{28} 31$ The present study has demonstrated how to adopt a broader societal perspective evaluating the economic efficiency of a public health intervention for railway injuries by exploring other outcome measurements and evaluation methods that are valid and relevant to this particular public health intervention.

The comparison of the two approaches of cost-effectiveness analysis by ICER demonstrates the impact of missing important outcomes on evaluating the economic efficiency of public health interventions. The analysis suggests that the use of DALYs alone, as the health outcome at the individual level from a victim perspective, is not sufficient to reflect a fair and appropriate value on the intervention's effectiveness from a societal perspective. Since everything is interconnected, nothing can be assumed constant from the consequence of intervention. ${ }^{28}$ Ignoring changes in other sectors would lead to an underestimation of the economic efficiency, which would adversely affect the advocacy of public health in the long run.

As shown in a previous study conducted by the authors, the presence of PSD has been proved to be effective for preventing suicides through means restriction. ${ }^{2} 4445$ However, due to its huge installation cost, it has been difficult in persuading the railway operator to extend the coverage of PSD to all railway stations in Hong Kong. ${ }^{2}$ It is believed that the findings of the present study can contribute to a more comprehensive framework in understanding on the potential social benefits from interventions, which is important for initiating other railway operators worldwide to follow.

These arguments have been asserted and recommended by the Toronto Transit Commission, ${ }^{46}$ which plans to begin the process of adding screen doors at select subway stations on the Yonge line beginning in 2013 and completion on the Yonge-UniversitySpadina line by $2015 .{ }^{47}$ However, as a consequence of a high installation cost of $\mathrm{C} \$ 10$ million per station, there remains no plan for the operator, which anticipates a $\mathrm{C} \$ 1.5$ billion capital shortfall by the end of the decade, ${ }^{47}$ to extend the coverage of PSD installation further to other stations. We expect the present study can provide some insights for every stakeholder to evaluate, if the work can bring any benefit to the society.

Several caveats of this analysis should be mentioned. First, we need to recognise that the use of a cost-effectiveness analysis is only one of the key elements for policymakers' consideration for resource allocation. Other concerns, such as availability of funds, media influence and community acceptance, would also affect the final decision. ${ }^{43}$ The present study illustrates only one of these critical inputs for the decision-making process. Second, as the geographical coverages of the two railway networks were not exactly identical, it is possible that the decrease in the number of casualties in MTRCL (or no decrease in KCRC) was partly due to some unknown exogenous factors, rather than the installation of PSDs. Third, as noted, the impact of risk at the platform edge interface is generally constructed by three dimensions, which includes the loss of human lives and functioning, passenger time due to the collapse of the railway service and also the corresponding psychological trauma following the incidence. However, in the present study, we have excluded the last item because we have no reliable information for quanti- fying the changes in psychological well-being following an incidence. The cost could be enormous.

More importantly, the use of DALY on cost-effectiveness analysis remains a highly controversial issue, in which not everyone appreciates the ethical dimension of the metric as the sole health outcome. ${ }^{48}$ The main disadvantage of focusing only on the time-base measure is that such an approach diverts attention away from the health needs of older adults and could be seen as discrimination on the basis of age. ${ }^{49}$ In figuring out a more comprehensive estimate on the health effect from intervention, more information would be needed about the circumstances of individuals who experience ill health (eg, the support provided through public services, private incomes, family, friends and so on). ${ }^{48}$

To conclude, the use of cost-effectiveness analysis allows decision-makers to target its resources on improving health and related issues more efficiently. Rather than worrying about the uncontrollable bias from the discretion nature of the railway system, public health researchers should spend more effort to recognise the nature and magnitude of these effects, and to

\section{What is already known on this subject}

- Railway injury has been considered an important public health issue, as each incident often produces ripple effects by causing psychological trauma in those who have witnessed the incident and causing breakdowns in the railway circulation. To prevent falls onto railway tracks (intentionally or accidentally), setting physical barriers, such as PSDs, is found to be effective, but its cost-effectiveness is not known.

- The importance of taking a societal perspective has been noted for economic assessments, but current cost-effectiveness studies have been focusing on measuring health changes at the individual level and summing them up to determine the societal effect, which may have omitted other important nonhealth outcomes and thus fails to give a reliable estimate of the cost-effectiveness of public preventive measures.

\section{What this study adds}

- This study shows that the presence of PSDs has effectively reduced railway injuries by an adjusted 5-year APC of $68.8 \%$ $(p<0.0001)$. The change did not appear to increase the risk to the other unsealed railway platforms, as the corresponding figure did not rise significantly $(p=0.5688)$.

- This study demonstrates how to include non-health factors as outcome measures. When DALY, loss of fare revenue and passenger waiting time are taken into consideration, the PSD installation is found to be cost-effective, with an ICER US $\$ 65400$ per life-year which is 2.62 times of per capita GDP in Hong Kong. The estimation based on health outcome (DALY) only would result in an ICER of US\$77 900 per life-year or 3.13 times the per capita GDP, which would lead to an underestimation of the cost-effectiveness. If a fair and appropriate evaluation of the intervention's cost-effectiveness from a societal perspective is not obtained, the advocacy of public health in the long run would be adversely affected. 
develop a more comprehensive approach and procedures of evaluating cost-effectiveness with new ways of thinking.

Acknowledgements The authors would like to thank the Mass Transit Railway Corporation Limited and the Transport and Housing Bureau of the Hong Kong Special Administrative Region government for providing the data necessary for our analysis.

Competing interests None.

Ethics approval Ethics approval was provided by the Human Research Ethics Committee for Non-Clinical Faculties of the University of Hong Kong.

Contributors CKL (the leading author) designed the study, formulated the research questions, undertook statistical analyses with PSFY and wrote the first draft of the manuscript.

Provenance and peer review Not commissioned; externally peer reviewed.

\section{REFERENCES}

1. Baumert J, Erazo N, Ladwig KH. Ten-year incidence and time trends of railway suicides in Germany from 1991 to 2000. Eur J Public Health 2005:16:173-8.

2. Law CK, Yip PS, Chan WS, et al. Evaluating the effectiveness of barrier installation for preventing railway suicides in Hong Kong. J Affect Disord 2009;114:254-62.

3. Lindekilde K, Wang AG. Train suicide in the county of FYN 1979-82. Acta Psychiatr Scand 1985;72:150-4.

4. O'Donnell I, Farmer RDT. Suicidal acts on metro systems: an international perspective. Acta Psychiatr Scand 1992;86:60-3.

5. Clarke M. Railway suicide in England and Wales, 1850-1949. Soc Sci Med 1994:38:401-7.

6. Clarke RV, Poyner B. Preventing suicide on the London underground. Soc Sci Med 1994;38:443-6.

7. Beskow J, Thorson J, Öström M. National suicide prevention programme and railway suicide. Soc Sci Med 1994;38:447-51.

8. Coats TJ, Walter DP. Effect of station design on death in the London underground: observational study. BMJ 1999:319:957.

9. Mishara BL. Railway and metro suicides: understanding the problem and prevention potential. Crisis 2007;28(Suppl 1):36-43

10. Neumann PJ. Why don't Americans use cost-effectiveness analysis? Am J Manag Care 2004;10:308-12.

11. You JH, Wong WC, Ip M, et al. Cost-effectiveness analysis of influenza and pneumococcal vaccination for Hong Kong elderly in long-term care facilities. $J$ Epidemiol Community Health 2009;63:906-11.

12. Tunis SR. Economic analysis in health decisions. Am J Manag Care 2004;10:301-4.

13. Neumann PJ, Rosen AB, Weinstein MC. Medicare and cost effectiveness analysis. N Engl J Med 2005;353:1516-22

14. Prosser LA, Koplan JP, Neumann PJ, et al. Barriers to using cost effectiveness analysis in managed care decision making. Am J Manag Care 2000;6:173-9.

15. Berger ML. The once and future application of cost-effectiveness analysis. Jt Comm J Qual Improv 1999:25:455-61.

16. Woo PP, Kim JJ, Leung GM. What is the most cost-effective population-based cancer screening program for Chinese women? J Clin Oncol 2007;25:617-24.

17. Murray CJL, Evans DB, Acharya A, et al. Development of WHO guidelines on generalized cost-effectiveness analysis. Health Econ 2000;9:235-51.

18. Evans DB, Adam T, Tan-Torres Edejer T, et al. Achieving the millennium development goals for health: Time to reassess strategies for improving health in developing countries. BMJ 2005;331:1133-6.

19. Evans DB, Tan-Torres Edejer T, Adam T, et al. Achieving the millennium development goals for health: methods to assess the costs and health effects of interventions for improving health in developing countries. BMJ 2005:331:1137-40.

20. Evans DB, Lim SS, Adam T, et al. Achieving the millennium development goals for health: evaluation of current strategies and future priorities for improving health in developing countries. BMJ 2005;331:1457-61.

21. Adam T, Lim SS, Mehta S, et al. Achieving the millennium development goals for health: cost effectiveness analysis of strategies for maternal and neonatal health in developing countries. BMJ 2005;331:1107.
22. Tan-Torres Edejer T, Aikins M, Black R, et al. Achieving the millennium development goals for health: Cost effectiveness analysisi of strategies for child health in develoiping countries. BMJ 2005;331:1177.

23. Morel CM, Lauer JA, Evans DB. Achieving the millennium development goals for health: Cost effectiveness analysis of strategies to combat malaria in developing countries. BMJ 2005;331:1299-302.

24. Baltussen R, Floyd K, Dye C. Achieving the millennium development goals for health: Cost effectiveness analysis of strategies for tuberculosis control in developing countries. BMJ 2005:331:1364.

25. Hogan DR, Baltussen R, Hayashi $C$, et al. Achieving the millennium development goals for health: Cost effectiveness analysis of strategies to combat HIV/AIDS in developing countries. BMJ 2005:331:1431-7.

26. Byford S, Raftery J. Perspectives in economic evaluation. BMJ 1998;316:1529-30

27. Weatherly H, Drummond M, Claxton K, et al. Methods for assessing the costeffectiveness of public health interventions: Key challenges and recommendations. Health Policy 2009;93:85-92.

28. Shiell A, Hawe P, Gold L. Complex interventions or complex systems? Implications for health economic evaluation. BMJ 2008;336:1281-3.

29. Hawe $\mathbf{P}$, Shiell A, Riley T. Complex interventions how out of control can a randomised controlled trial be? BMJ 2004;328:1561-3.

30. Schmidtke A. Suicidal behaviour on railway in the FRG. Soc Sci Med 1994;38:419-26

31. Sagoff M. Policy analysis and social values. In: Carrow MM, Churchill RP, Cordes J J J eds. Democracy, Social Values, and Public Policy. Westport, CT: Greenwood Publishers, 1998:91-106.

32. World Health Organization. The Ninth Revision of the International Classification of Diseases and Related Health Problems. 9th edn. Geneva: WHO, 1979

33. World Health Organization. The Tenth Revision of the International Classification of Diseases and Related Health Problems. 10th edn. Geneva: WHO, 1992

34. Tong D. Platform screen doors programme of MTR Corporation-better environment for less energy. Proceedings of the First International Conference on Energy Efficiency and Conservation, Hong Kong, 2003:74-7.

35. Murray CJL. Rethinking DALYs. In: Murray CJL, Lopez AD, eds. The Global Burden of Disease: A Comprehensive Assessment of Mortality and Disability From Diseases, Injuries and Risk Factors in 1990 and Projected to 2020. Cambridge, MA: Harvard School of Public Health, 1996:1-98.

36. Murray CJL, Acharya AK. Understanding DALYs. J Health Econ 1997;16:703-30.

37. World Health Organization. Macroeconomics and Health: Investing in Health for Economic Development. The Global Burden of Disease 2004 Update. Geneva: WHO 2008.

38. Mass Transit Railway Corporation Limited. Platform screen doors. http://www mtr.com.hk/eng/sustainability/sustainrpt/2005rpt/sia-psd.html laccessed 13 Apr 2010)

39. Hong Kong Institute of Certified Public Accountants. Hong Kong Accounting Standard 11 Construction Contracts, http://www.hkicpa.org.hk/ebook/HKSA Members_Handbook_Master/volumell/hkas11.pdf (accessed on 12 Jan 2009)

40. Mass Transit Railway Corporation Limited. 2007 Annual Report: A New Era Hong Kong: MTRCL, 2008

41. Bonneux $\mathbf{L}$, Birnie E. The discount rate in the economic evaluation of prevention: a thought experiment. J Epidemiol Community Health 2001;55:123-5.

42. Franco $\mathbf{O H}$, Steyerberg EW, de Laet $\mathrm{C}$. The polypill: at what price would it become cost effective? J Epidemiol Community Health 2006;60:213-17.

43. Murray CJL, Lauer JA, Hutubessy RC, et al. Effectiveness and costs of interventions to lower systolic blood pressure and cholesterol: a global and regional analysis on reduction of cardiovascular-disease risk. Lancet 2003;361:717-25.

44. Mann JJ, Apter A, Bertolote J, et al. Suicide prevention strategies: a systematic review. JAMA 2005;294:2064-74

45. Yip PSF, Law CK, Fu KW, et al. Restricting the means of suicide by charcoal burning Br J Psychiatry 2010;196:241-2.

46. Toronto Transit Commission. Subway Suicide Prevention. Toronto: TTC, 2010

47. Thestar.com. TTC plans suicide barriers on Yonge line. http://www.thestar.com/ news/gta/transportation/article/784800-ttc-plans-suicide-barriers-on-yonge-line? bn=1 (accessed 15 Oct 2010).

48. Anand S, Hanson K. Disability-adjusted life years: a critical review. J Health Econ 1997; 16:685-702.

49. Gunnell D, Middleton N. National suicide rates as an indicator of the effect of suicide on premature mortality. Lancet 2003:362:961-2. 